\title{
Coupling and proximity effects in the superfluid transition in ${ }^{4} \mathrm{He}$ dots
}

\author{
Justin K. Perron, Mark O. Kimball, Kevin P. Mooney and Francis M. Gasparini ${ }^{\star}$
}

The superfluid transition in ${ }^{4} \mathrm{He}$ is associated with the onset of an order parameter that is a macroscopic wavefunction. This is similar to the onset of superconductivity in metals and alloys. Yet, some of the hallmark phenomena that are associated with wavefunctions, and are evident in superconductors, have not been identified in ${ }^{4} \mathrm{He}$. Here we report proximity effects on the specific heat and superfluid density of a thin ${ }^{4} \mathrm{He}$ film in equilibrium with an array of larger boxes of ${ }^{4} \mathrm{He}$. Comparison with previous data also enables us, by scaling, to deduce an excess specific heat associated with a collective behaviour of weakly coupled boxes. These effects should be relevant to the understanding of experiments where helium has been studied when confined in packed powders, porous materials or other inhomogeneous confinements, where proximity and collective effects must be present but not readily quantifiable ${ }^{1,2}$. Our work also forms a point of comparison and contrast with the case of superconductors ${ }^{3,4}$, and is relevant to studies of Josephson effects in superfluids, coupling effects in arrays of Bose-condensed atoms ${ }^{5,6}$, and magnetic systems where, because of chemical composition, two separate but interacting ordering transitions take place ${ }^{7,8}$.

In the case of superconductors, coupling and proximity effects have been well understood for many years. The large correlationlength amplitude $\xi_{0}$ makes coupling between two superconductors easy to achieve. Weak links with dimensions of the order of $\xi_{0}$, which can be a metal, an insulator or another superconductor, enable measurements of the familiar Josephson effects (see for instance ref. 9). With ${ }^{4} \mathrm{He}$ these effects are much more difficult to achieve because $\xi_{0}$ is of the order of interatomic dimensions. Furthermore, weak links and coupling between two regions of ${ }^{4} \mathrm{He}$ cannot be achieved through any material other than ${ }^{4} \mathrm{He}$. We can, however, take advantage of the growth of the correlation length with temperature $T$ and critical exponent $v, \xi=\xi_{0}\left|1-T / T_{\lambda}\right|^{-v} \equiv \xi_{0} t^{-v}$ near the transition temperature $T_{\lambda}$ and construct apertures whereby analogous Josephson effects can be realized ${ }^{10,11}$.

We are unaware of any theory that describes the effects on thermodynamic properties of a 'grain' or dot of helium due to a coupling to another dot through a weak link, or, for that matter, how this coupling is affected by the geometry or 'strength' of this link. This question arose while attempting to verify finite-size scaling for zero-dimensional crossover ${ }^{12}$. We had measured the heat capacity of helium in arrays of $(1 \mu \mathrm{m})^{3}$ and $(2 \mu \mathrm{m})^{3}$ boxes linked by channels of $1 \mu \mathrm{m} \times 1 \mu \mathrm{m} \times 0.019 \mu \mathrm{m}$ and $2 \mu \mathrm{m} \times 2 \mu \mathrm{m} \times 0.010 \mu \mathrm{m}$ respectively. These data did not scale anywhere in the critical region $^{13}$. The lack of scaling is unexpected, because for both twoand one-dimensional crossover, at least for $T>T_{\lambda}$, other data do scale $^{14}$. More significantly, an analysis of the zero-dimensional data suggested that the smaller boxes had a higher heat capacity than expected ${ }^{13}$. This might result from a coupling among these boxes, which, before the present work, had been an open question.

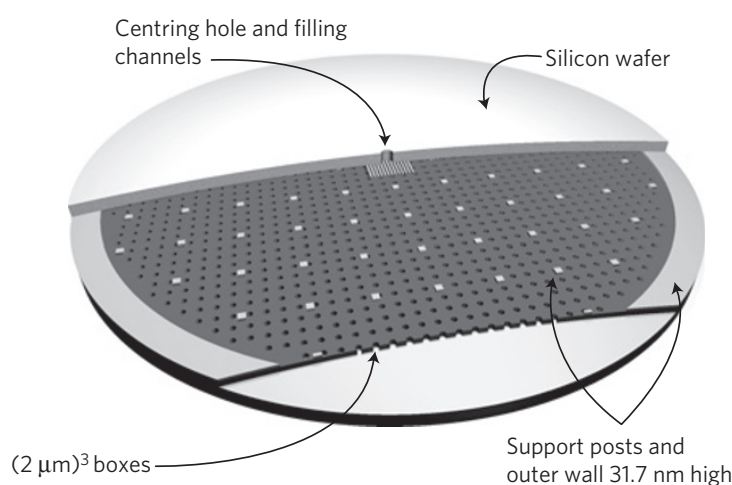

Figure 1 | The confinement cell. A cut-away view, not to scale, of a cell of $(2 \mu \mathrm{m})^{3}$ boxes connected by a $31.7 \mathrm{~nm}$ planar region. This planar region is formed when the top wafer is bonded to the bottom wafer at the separation determined by the support posts and outer wall. This cell consists of two oxidized and patterned wafers of silicon $5 \mathrm{~cm}$ in diameter. One wafer has an array of $3.4 \times 10^{7}$ boxes of $(2 \mu \mathrm{m})^{3}$ volume patterned in the $\mathrm{SiO}_{2}$. The top wafer has a series of square support posts formed from a $(31.7 \pm 0.1) \mathrm{nm}$ oxide growth. The centre of the top wafer has a series of 8-mm-long, $15-\mu \mathrm{m}$-wide channels that take the helium from the centre filling hole to the rest of the cell. The cell is formed by direct wafer bonding ${ }^{15}$. The resulting structure is an array of boxes spaced $6 \mu \mathrm{m}$ centre to centre and connected through a planar opening $31.7 \mathrm{~nm}$ high.

The experimental cell used in the present study is described and shown schematically in Fig. 1 . The cell confines ${ }^{4} \mathrm{He}$ to a array of $(2 \mu \mathrm{m})^{3}$ boxes, $6 \mu \mathrm{m}$ centre to centre, with a $31.7 \pm 0.1 \mathrm{~nm}{ }^{4} \mathrm{He}$ film linking them. The uncertainty in the film thickness is the standard deviation associated with the measurement of the thermal oxide before the patterning of this oxide into the posts and wall shown in Fig. 1. The rationale for the design at the centre of the cell and the eventual staging of the cell to measure heat capacity have been discussed previously ${ }^{16}$. The superfluid density $\rho_{\mathrm{s}}$ of the film is obtained by using adiabatic fountain resonance ${ }^{17}$.

The heat capacity of ${ }^{4} \mathrm{He}$ confined in this cell is shown as filled circles in Fig. 2. These data are for $T<T_{\lambda}$ (upper branch) and $T>T_{\lambda}$ (lower branch). The dominant peak near $t \cong 1.5 \times 10^{-5} t_{\mathrm{m}, \mathrm{b}}$ is the maximum for the transition of ${ }^{4} \mathrm{He}$ confined in the boxes. It is shifted below $T_{\lambda}$ as expected from finite-size effects ${ }^{18}$. Because the connecting film is normal at this temperature, the order parameter in each box has an arbitrary phase. The second feature at $t \cong 1.4 \times 10^{-3} t_{\mathrm{m}, \mathrm{f}}$ is associated with the transition in the $31.7 \mathrm{~nm}$ film. The superfluid onset for the film (see below) takes place at a yet lower temperature $t_{\mathrm{c}}$. It is at $t_{\mathrm{c}}$ that a global phase coherence must be established. However, because of the confinement, the magnitude of $\rho_{\mathrm{s}}$ will be different in the boxes and the film. This observation of a two-step transition into global coherence 


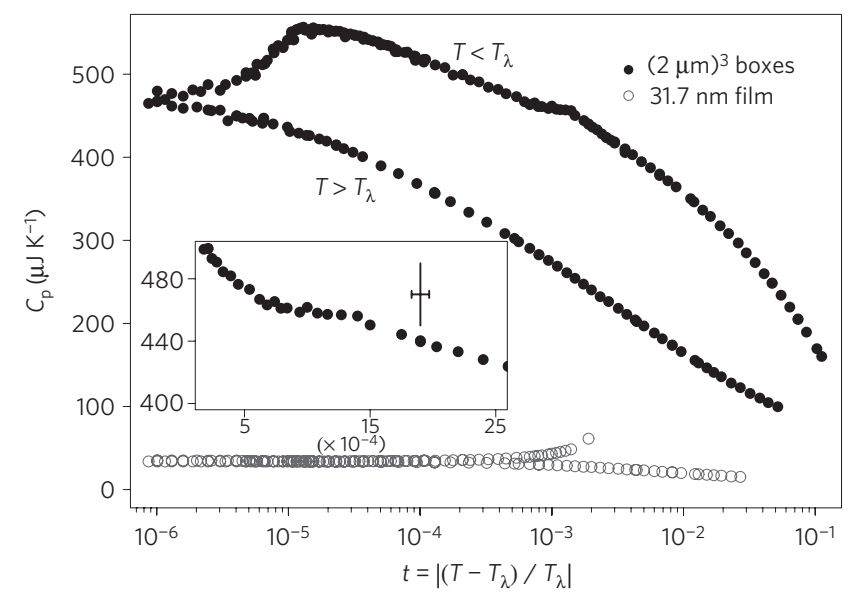

Figure 2 | Heat-capacity signatures of a two-step transition. The total measured heat capacity for the helium in the cell described in Fig. 1 (filled circles), and the calculated heat capacity for a $31.7 \mathrm{~nm}$ uniform film (open circles). This calculation is done using the scaling functions for planar confinement, Equations (1) and (2), and using $1.22 \mu \mathrm{mol}$ for the amount of helium in the film. The inset shows an expanded view of the data in the region where the film orders. Here the vertical line indicates the expected temperature $T_{m}$ of the specific heat maximum if the film were not influenced by the presence of boxes ${ }^{14}$. This is obtained by a least-squares fit of the data for planar confinement ${ }^{14}$ and yields $t_{\mathrm{m}}(31.7 \mathrm{~nm})=(1.90 \pm 0.07) \times 10^{-3}$.

for ${ }^{4} \mathrm{He}$ mirrors observations with granular superconductors ${ }^{4,19}$, and superconducting $\operatorname{arrays}^{20}$. This was also observed earlier in a less-well-controlled geometry, where proximity and coupling effects could not be identified ${ }^{21}$. We also note that calculations of the specific heat of coupled regions of two-dimensional Ising spins result in a two-peak structure: one associated with local ordering and another with long-range order ${ }^{22}$.

In the inset of Fig. 2 we indicate with a vertical line the expected position of the specific-heat maximum for a $31.7 \mathrm{~nm}$ planar film without the influence of the boxes. This is well known from measurements over a wide range of film thickness ${ }^{14}$. The signature of the film's maximum in the present data is shifted, $\delta t_{\mathrm{m}, \mathrm{f}} \cong(0.5 \pm 0.07) \times 10^{-3}$ warmer than expected, an indication of the influence of the already superfluid boxes on the heat-capacity maximum of the normal film. From this observation we conclude that the boxes and film, at least near $t_{\mathrm{m}, \mathrm{f}}$, act as a collective, rather than separate, thermodynamic system. To see the full extent of this behaviour, we can subtract from the total heat capacity that of a $31.7 \mathrm{~nm}$ uniform film, which can be calculated using empirical scaling functions established for films in other studies ${ }^{14}$. Finitesize correlation-length scaling ${ }^{18}$ predicts that the thermodynamic response of a finite system of small dimension $L$ should be related to its behaviour in the thermodynamic limit by functions that depend on the variable $L / \xi$, or, equivalently, after introducing $\xi=\xi_{0} t^{-\nu}$, the variable $t l^{1 / v}$ with $l \equiv L / \xi_{0}, \xi_{0}=0.143 \mathrm{~nm}$ for ${ }^{4} \mathrm{He}$ and $T>T_{\lambda}$. In the case of the specific heat, a convenient function to use is the difference at the same temperature between the specific heat in the thermodynamic limit $C(t, \infty)$ and that of the finite system $C(t, L)$. This difference should be related to a scaling function $g_{1}\left(t l^{1 / v}\right)$ as ${ }^{14}$

$$
[C(t, \infty)-C(t, l)] t^{\alpha} \equiv \Delta C t^{\alpha}=g_{1}\left(t l^{1 / v}\right)
$$

where the exponents $v=0.6705$ (ref. 23) and $\alpha=-0.0115$ are consistent with the hyperscaling relation $\alpha=2-3 v$. Measurements for films over a wide range of $L$ support this expected scaling behaviour up to the neighbourhood of the specific-heat maximum. Thus the specific heat of any film can be calculated from the scaling

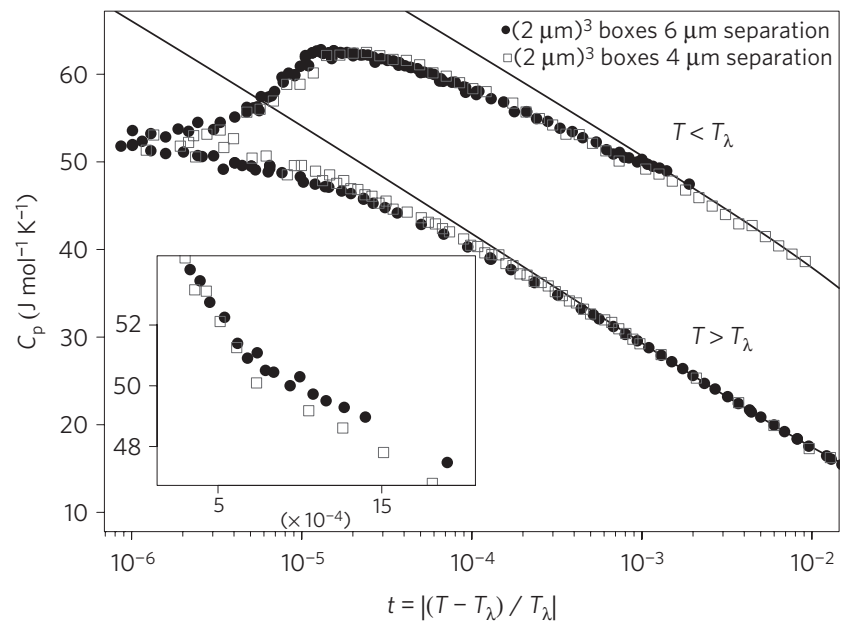

Figure 3 | Isolated boxes and enhanced film. The specific heat for helium confined in $(2 \mu \mathrm{m})^{3}$ boxes with very different connecting regions, and therefore different couplings, is plotted. For the filled circles the data represent that of Fig. 1 with the calculated contribution of a uniform $31.7 \mathrm{~nm}$ film subtracted. For the data shown as the open boxes such correction is not needed because the film contributes a negligible amount. The relatively good agreement between these two sets of data in most of the temperature region indicates that they represent the specific heat of isolated $(2 \mu \mathrm{m})^{3}$ boxes of helium. The inset is an expanded view of the region where the $31.7 \mathrm{~nm}$ film becomes superfluid. The calculated contribution of a uniform film in this region still leaves an excess specific heat, which is an enhancement owing to the proximity of the film to the helium in the boxes An analogous effect of proximity will be seen in the superfluid density in Fig. 4. The solid lines represent the behaviour of bulk helium.

function $g_{1}\left(t l^{1 / v}\right)$. For temperatures above $T_{\lambda}$ we can represent $g_{1}$ with the function ${ }^{24}$

$$
g_{1}^{+}(x)=\frac{A / \alpha}{1+a x^{v}}+\frac{b x^{\alpha}}{1+c x^{\alpha+v}}
$$

where the fitting parameters are given in refs 14 and 24. Similarly, below $T_{\lambda}$, and up to $x \cong 4.7$, we can use ${ }^{13}$

$$
g_{1}^{-}(x)=A^{-} \ln x+b^{-} x^{2}+c^{-} x+d
$$

This function, with parameters in $\mathrm{J} \mathrm{mol}^{-1} \mathrm{~K}^{-1} A^{-}=-45.72 \pm 0.18$, $b^{-}=-0.3191 \pm 0.053, c^{-}=-1.244 \pm 0.028, d^{-}=31.21 \pm 0.25$, fits the scaled planar data with random deviations in the region

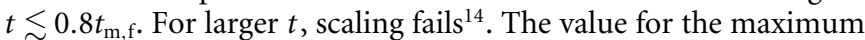
can be deduced independently of equation (3) from existing data ${ }^{14}$. The calculated heat capacity of a $1.22 \mu \mathrm{mol}$ uniform film $31.7 \mathrm{~nm}$ thick is shown at the bottom of Fig. 2 as open circles. This can be subtracted from the total heat capacity to obtain the specific heat of the helium in the boxes. These data are shown as the filled circles in Fig. 3. The solid lines are $C(t, \infty)$ (ref. 16), and our data have been normalized to this in the region $t \geq 10^{-3}\left(T>T_{\lambda}\right)$. Also on this plot, we show the specific heat from an earlier set of measurements where the $(2 \mu \mathrm{m})^{3}$ boxes were spaced $4 \mu \mathrm{m}$ centre to centre and were connected with channels of $2 \mu \mathrm{m} \times 2 \mu \mathrm{m} \times 0.010 \mu \mathrm{m}$ (ref. 12). In contrast to the present cell, the helium in these 10 -nm-high connecting channels represents only $0.5 \%$ of the helium in the cell and contributes negligibly to the heat capacity. The good agreement between the two sets of data in Fig. 3 (apart from the region where the film orders) leads to the conclusion that, in these two rather different arrangements of the boxes, the data represent very nearly the specific heat of isolated $(2 \mu \mathrm{m})^{3}$ dots of helium.

Shown in the inset of Fig. 3 is the region where the film orders. The calculated subtraction for the contribution of a uniform film 


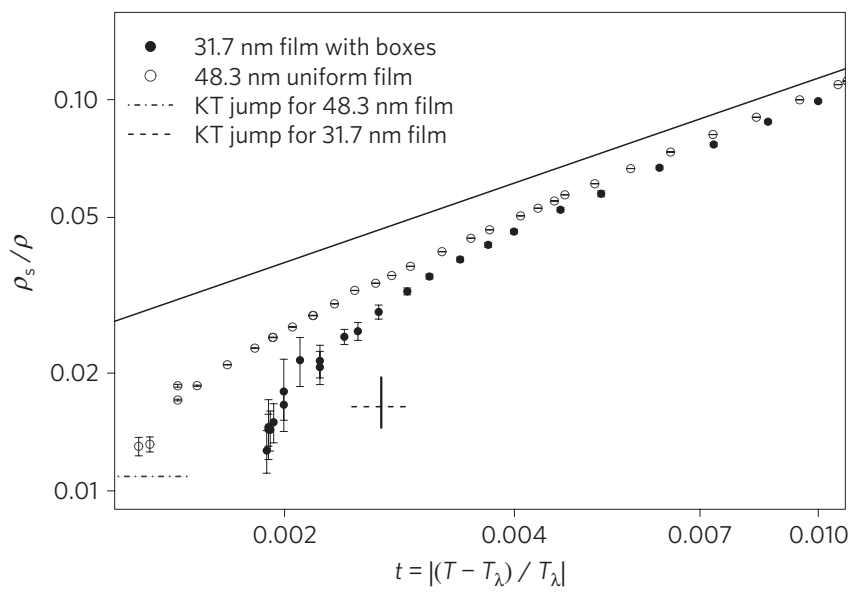

Figure 4 | Enhanced superfluid onset and superfluid fraction. Plot of the superfluid fraction for a uniform $48.3 \mathrm{~nm}$ film (open circles) and a $31.7 \mathrm{~nm}$ film in the presence of helium in $(2 \mu \mathrm{m})^{3}$ boxes at $6 \mu \mathrm{m}$ separation centre to centre (filled circles). The solid line is the bulk behaviour. The horizontal lines represent the lowest values of $\rho_{\mathrm{s}} / \rho$ expected for uniform films of the respective sizes ${ }^{28}$. The solid vertical line is at the expected $t_{c}$ for a uniform $31.7 \mathrm{~nm}$ film. This is obtained by a least-squares fit of the data for planar confinement and yields $t_{\mathrm{c}}(31.7 \mathrm{~nm})=(2.75 \pm 0.04) \times 10^{-3}$. We can see that the film in the presence of the boxes has $\rho_{\mathrm{s}} / \rho$ that persists to a higher temperature than expected and to a lower value than expected. If these enhancement effects were not present, the filled circles should end at the point where the vertical line and horizontal dashed line cross. The vertical error bars are standard deviations associated with the determination of the resonance frequency from which $\rho_{\mathrm{s}} / \rho$ is obtained.

leaves the data above those for the $(2 \mu \mathrm{m})^{3}$ boxes unaffected by a film. This indicates that the film correction, using the scaling function, as well as the individual point for the film's maximum, underestimates the contribution of the $31.7 \mathrm{~nm}$ film, or, equivalently, that the specific heat of the film in the presence of the superfluid boxes has been enhanced.

Just as the specific heat of the film is affected by the boxes, so is the superfluid fraction $\rho_{\mathrm{s}} / \rho$, which is related to the order parameter. To measure this we drive a resonant superflow in the film, which takes place over a 'bed' of already superfluid boxes. This flow is driven thermally, thus causing superfluid to move in and out of the cell. The restoring force comes from the compressibility of the helium. The peak mass flow is typically $\sim 10^{-12} \mathrm{~g} \mathrm{~m}$ with temperature excursions of less than $10^{-6} \mathrm{~K}$. Details of the equations of resonance and the hydrodynamic analysis can be found in ref. 17. Data for $\rho_{\mathrm{s}} / \rho$ are shown in Fig. 4. Two sets of data are plotted here. The open circles are for a uniform film $48.3 \mathrm{~nm}$ thick ${ }^{25}$, and the filled circles are for the $31.7 \mathrm{~nm}$ film in the presence of the $(2 \mu \mathrm{m})^{3}$ boxes. The behaviour of the bulk $\rho_{\mathrm{s}} / \rho$ is indicated as a solid line ${ }^{26}$. The superfluid onset for the uniform $48.3 \mathrm{~nm}$ film is consistent with the $t_{\mathrm{c}}$ in other uniform films ${ }^{14}$. The smallest value in $\rho_{\mathrm{s}} / \rho$ at the transition is also consistent with, but somewhat larger $(\sim 20 \%)$ than, the expected Kosterlitz-Thouless (KT) jump ${ }^{27,28}$ (indicated by the dashed-dotted horizontal line). For a uniform $31.7 \mathrm{~nm}$ film the expected position of $t_{\mathrm{c}}$ and expected KT jump are indicated with the crossed solid vertical and dashed horizontal lines respectively. It is at this crossing point that $\rho_{\mathrm{s}} / \rho$ is expected to vanish for this film. We can see that the presence of the boxes has caused $\rho_{\mathrm{s}} / \rho$ to vanish at a higher temperature than expected, and with a lower magnitude than the expected KT jump. In none of our previous measurements using adiabatic fountain resonance have we measured a smaller than expected jump in $\rho_{\mathrm{s}} / \rho$. Rather, as seen in the $48.3 \mathrm{~nm}$ data, measurements will yield a somewhat larger jump simply because, owing to dissipation, the resonance cannot

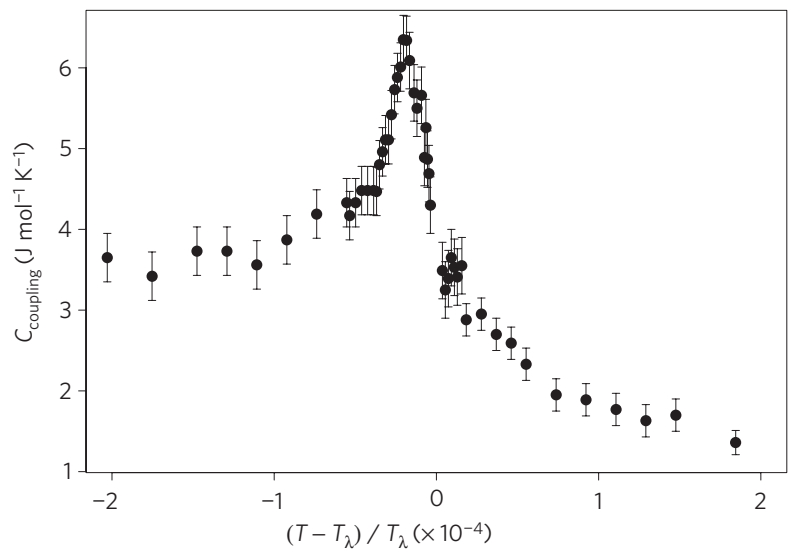

Figure 5 | Specific heat associated with coupling of helium confined to $(\mathbf{1} \boldsymbol{\mu m})^{\mathbf{3}}$ boxes. The excess specific heat $C_{\text {coupling }}$ is obtained using equation (4). The $(1 \mu \mathrm{m})^{3}$ boxes are separated at $2 \mu \mathrm{m}$ centre to centre and are linked with a channel $1 \mu \mathrm{m}$ wide and $19 \mathrm{~nm}$ high. As noted in the text, the coupling between these boxes extends well into regions where $\xi \ll 1 \mu \mathrm{m}$, which is the edge-to-edge separation of the boxes. The error bars for the data represent the combined uncertainty in the value of the experimentally determined functions $\left.g_{1}(x)\right|_{2 \mu \mathrm{m}}$ and $\left.g_{1}(x)\right|_{1 \mu \mathrm{m}}$; see equation (4) and Supplementary Information.

be followed too close to $t_{\mathrm{c}}$. The enhancement of $\rho_{\mathrm{s}} / \rho$ for confined helium in the presence of bulk liquid has been derived using a mean-field approach ${ }^{29}$. As the helium in the boxes can be viewed as nearly bulk at the temperature where the film orders, we view this as qualitative support for our observations of the behaviour of the film in the presence of the boxes.

We identify collectively the shift to higher temperature of $t_{\mathrm{m}, \mathrm{f}}$ and $t_{\mathrm{c}}$ and the enhancement of both the specific heat (Fig. 3) and $\rho_{\mathrm{s}} / \rho$ (Fig. 4) as proximity effects on the $31.7 \mathrm{~nm}$ film in the presence of the helium in the $(2 \mu \mathrm{m})^{3}$ boxes.

Returning now to the discussion of the zero-dimensional scaling, we can conclude, from the general agreement between the two sets of $(2 \mu \mathrm{m})^{3}$ data in Fig. 3, obtained under different conditions of spacing and connectivity, that the previously observed lack of scaling between the $(2 \mu \mathrm{m})^{3}$ and $(1 \mu \mathrm{m})^{3}$ boxes data is due to the behaviour of the latter. This lack of scaling is manifest in the fact that at the same value of the scaling variable $x=t l^{1 / v}$ the measured quantities on the left-hand side of equation (1) produce two different values for $g_{1}(x):\left.g_{1}(x)\right|_{2 \mu \mathrm{m}}$ and $\left.g_{1}(x)\right|_{1 \mu \mathrm{m}}$. This can be seen in Fig. 47 of ref. 14, and is provided as Supplementary Information. For the $(1 \mu \mathrm{m})^{3}$ data we can separate in equation (1) the coupling contribution from the scaling part, or

$$
\Delta C=\Delta C_{\text {scaling }}+C_{\text {coupling }}
$$

This yields from equation (1)

$$
C_{\text {coupling }}=t^{-\alpha}\left[\left.g_{1}(x)\right|_{2 \mu \mathrm{m}}-\left.g_{1}(x)\right|_{1 \mu \mathrm{m}}\right]
$$

This result is plotted in Fig. 5 for the region near $T_{\lambda}$. We can see that the coupling of the $(1 \mu \mathrm{m})^{3}$ boxes is significant: it amounts to a $10 \%$ increase over the total specific heat at the maximum. The position of the maximum in $C_{\text {coupling }}$ is at $t_{\mathrm{m}, \mathrm{Co}}=(2.0 \pm 0.5) \times 10^{-5}$. This is somewhat closer to $T_{\lambda}$ than the maximum of the total specific heat, which is at $t_{\mathrm{m}, \mathrm{b}}=(3.6 \pm 0.6) \times 10^{-5}$ (ref. 30$)$; see also Supplementary Information. The coupling of these boxes is surprising if we consider the fact that the correlation length is smaller than the separation of $1 \mu \mathrm{m}$ (at $t=10^{-4}$ the bulk correlation length is $0.07 \mu \mathrm{m}$ and $0.17 \mu \mathrm{m}$ above and below $T_{\lambda}$ respectively), and, as well, that the helium in the connecting channels does not 
become superfluid until $t_{\mathrm{c}} \cong 0.02$, about three decades colder ${ }^{31}$. Figure 5 represents the first identification of collective effects on the specific heat of weakly coupled regions of ${ }^{4} \mathrm{He}$ near the superfluid transition. The enhancement of the specific heat may be viewed as a partial crossover to two-dimensional behaviour as opposed to zero dimensional for fully isolated boxes. There is no equivalent observation to this with superconductors because in superconductors the specific heat is not detectably enhanced by critical fluctuations.

Received 5 November 2009; accepted 12 April 2010; published online 23 May 2010

\section{References}

1. Reppy, J. D. Superfluid helium in porous media. J. Low Temp. Phys. 87, 205-245 (1992).

2. Gasparini, F. M. \& Rhee, I. in Progress in Low Temperature Physics, Vol. 13 (ed. Brewer, D. F.) 1-90 (North Holland, 1992).

3. van der Zant, H. S. J., Elion, W. J., Geerlings, L. J. \& Mooij, J. E. Quantum phase transitions in two dimensions: Experiments in Josephson-junction arrays. Phys. Rev. B 54, 10081-10093 (1996).

4. Jaeger, H. M., Haviland, D. B., Orr, B. \& Goldman, A. M. Onset of superconductivity in ultrathin granular metal films. Phys. Rev. B 40, 182-196 (1989).

5. Bloch, I., Dalibard, J. \& Zwerger, W. Many-body physics with ultracold gases. Rev. Mod. Phys. 80, 885-964 (2008).

6. Gati, R., Hemmerling, B., Fölling, J., Albiez, M. \& Oberthaler, M. K. Noise thermometry with two weakly coupled Bose-Einstein condensates. Phys. Rev. Lett. 96, 130404 (2006).

7. Sobolev, V. L., Vitebsky, I. M. \& Lisyansky, A. A. Magnetic phase transition with final ordering: Peculiarities in the critical behavior. Phys. Rev. B 47, 8653-8659 (1993).

8. Ivanchenko, Y. M., Lisyanskii, A. A. \& Fillipov, A. E. Fluctuation effects in two-sublattice magnetic materials. Sov. Phys. Solid State 28, 608-611 (1986).

9. Tinkham, M. Introduction to Superconductivity (McGraw-Hill, 1996).

10. Sukhatme, K., Mukharsky, Y., Chui, T. \& Pearson, D. Observation of the ideal Josephson effect in superfluid ${ }^{4} \mathrm{He}$. Nature 411, 280-283 (2001).

11. Hoskinson, E., Sato, Y. \& Packard, R. E. Superfluid ${ }^{4} \mathrm{He}$ interferometer operating near 2 K. Phys. Rev. B 74, 100509 (2006).

12. Mooney, K. P. Confinement, Topology, Universality, and Non-scaling Effects in the Specific Heat and Superfluid Density of ${ }^{4} \mathrm{He}$ Near the Superfluid Transition. $\mathrm{PhD}$ thesis, Univ. Buffalo, The State University of New York (2006).

13. Perron, J. K., Kimball, M. O., Mooney, K. P. \& Gasparini, F. M. Lack of correlation-length scaling for an array of boxes. J. Phys. Chem. Solid 150, 032082-032086 (2009)

14. Gasparini, F. M., Kimball, M. O., Mooney, K. P. \& Diaz-Avila, M. Finite-size scaling of ${ }^{4} \mathrm{He}$ at the superfluid transition. Rev. Mod. Phys. 80, 1009-1059 (2008).

15. Rhee, I., Bishop, D. J., Petrou, A. \& Gasparini, F. M. Si wafers uniformly spaced; bonding and diagnostics. Rev. Sci. Instrum. 61, 1528-1536 (1990).

16. Mehta, S., Kimball, M. O. \& Gasparini, F. M. Superfluid transition of ${ }^{4} \mathrm{He}$ for two-dimensional crossover, heat capacity, and finite-size scaling. J. Low Temp. Phys. 114, 467-521 (1999).
17. Gasparini, F. M., Kimball, M. O. \& Mehta, S. Adiabatic fountain resonance for ${ }^{4} \mathrm{He}$ and ${ }^{3} \mathrm{He}-{ }^{4} \mathrm{He}$ mixtures. J. Low Temp. Phys. 125, 215-238 (2001).

18. Fisher, M. E. in Critical Phenomena Proc. 51st 'Enrico Fermi' Summer School, Varenna, Italy (ed. Green, M.) (Academic, 1971).

19. Costa, R. M., Ferreira, L. M., Vieira, V. N., Pureur, P. \& Schaf, J. Coherence transition in granular $\mathrm{YBa}_{2} \mathrm{Cu}_{3} \mathrm{O}_{7-\delta}, \mathrm{YBa}_{2} \mathrm{Cu}_{2.95} \mathrm{Zn}_{0.05} \mathrm{O}_{7-\delta}$, and $\mathrm{YBa}_{1.75} \mathrm{Sr}_{0.25} \mathrm{Cu}_{3} \mathrm{O}_{7-\delta}$ superconductors. Euro. Phys. J. B 58, 107-113 (2007).

20. Resnick, D. J., Garland, J. C., Boyd, J. T., Shoemaker, S. \& Newrock, R. S. Kosterlitz-Thouless transition in proximity-coupled superconducting arrays. Phys. Rev. Lett. 47, 1542-1545 (1981).

21. Chen, T. P., DiPirro, M. J., Gaeta, A. A. \& Gasparini, F. M. Adsorption and specific heat studies of ${ }^{4} \mathrm{He}$ in $0.2-\mu \mathrm{m}$ nuclepore filters. J. Low Temp. Phys. 26, 927-944 (1976).

22. Fisher, M. E. Aspects of equilibrium critical phenomena. J. Phys. Soc. Jpn (Suppl.) 26, 87-93 (1969).

23. Goldner, L. S., Mulders, N. \& Ahlers, G. Second sound very near $T_{\lambda}$. J. Low Temp. Phys. 93, 125-176 (1993).

24. Mehta, S. \& Gasparini, F. M. Specific heat and scaling of ${ }^{4} \mathrm{He}$ confined in a planar geometry. Phys. Rev. Lett. 78, 2596-2599 (1997).

25. Kimball, M. O. \& Gasparini, F. M. Superfluid fraction of ${ }^{3} \mathrm{He}-{ }^{4} \mathrm{He}$ mixtures confined at $0.0483 \mu \mathrm{m}$ between silicon wafers. Phys. Rev. Lett. 86, 1558-1561 (2001).

26. Greywall, D. S. \& Ahlers, G. Second-sound velocity, scaling, and universality in He-II under pressure near the superfluid transition. Phys. Rev. Lett. 28, 1251-1254 (1972).

27. Kosterlitz, J. M. \& Thouless, D. J. Ordering, metastability and phase transitions in two-dimensional systems. J. Phys. C 6, 1181-1203 (1973).

28. Nelson, D. R. \& Kosterlitz, J. M. Universal jump in the superfluid density of two-dimensional superfluids. Phys. Rev. Lett. 39, 1201-1205 (1977).

29. Mamaladze, Y. G. \& Cheishvili, O. D. Flow of a superfluid liquid in porous media. Sov. Phys. JETP 23, 112-117 (1967).

30. Kimball, M. O., Mooney, K. P. \& Gasparini, F. M. Three-dimensional critical behavior with 2D, 1D, and 0D dimensionality crossover: Surface and edge specific heats. Phys. Rev. Lett. 92, 115301 (2004).

31. Kimball, M. O. Helium Confined to a Restricted Geometry - A Study of Lower Dimensionality Crossover and Universality. $\mathrm{PhD}$ thesis, Univ. Buffalo, The State University of New York (2005).

\section{Acknowledgements}

This work is supported by the NSF, grant no. DMR-0605716; the Cornell Nanoscale Science and Technology Facility, project no. 526-94, and internal funding from the University at Buffalo.

\section{Author contributions}

F.M.G. proposed the experiment. M.O.K. and J.K.P. built the $(2 \mu \mathrm{m})^{3}$ confinement cell with the $31.7 \mathrm{~nm}$ planar connecting region and collected the data for that cell. K.P.M. and M.O.K. built and measured the previous cells and analysed their data. The analysis of $C_{\text {coupling }}$, the KT jump and shifts in $t_{\mathrm{c}}$ and $T_{\mathrm{m}, \mathrm{f}}$ were carried out by J.K.P. and F.M.G.

\section{Additional information}

The authors declare no competing financial interests. Supplementary information accompanies this paper on www.nature.com/naturephysics. Reprints and permissions information is available online at http://npg.nature.com/reprintsandpermissions. Correspondence and requests for materials should be addressed to F.M.G. 